\title{
Two Port Laparoscopic Cholecystectomy-An Initial Experience of 25 Cases with a New Technique
}

\author{
Aswini Misra A
}

\section{ABSTRACT}

Background: In Nepal, it is quite common to find patients with large stone burden and thick gallbladderwall which often leads to incision extension. We have used this extended incision to our advantage.The present technique of 2 port Laparoscopic cholecystectomy not only helps overcoming thespecimen extraction difficulties but also contributes to better cosmesis.

Patients and methods: Total of 25 patients were underwent the surgery in 2008-2010.

Results: The mean operating time was 50 minutes. None had significant procedural blood loss, iatrogenic injury, perforation of gallbladder, bile spillage, significant gas leak or subcutaneousemphysema at either port site. All patients were comfortable in the postoperative period and were routinely discharged on 2nd postoperative day except for 2 patients who has surgical site infection and fever respectively. Although 3 cases were converted to standard 4 port technique, none required conversion to open cholecystectomy. Out of 25 patients, 7 cases have completed 3 months follow up and did not show any complication like port site hernia.

Conclusion: The described method of performing 2 port laparoscopic cholecystectomy is safe,simple and inexpensive yet cosmetically rewarding.

Keywords: Cholecystectomy, Laparoscopic.

How to cite this article: Misra AA. Two Port Laparoscopic Cholecystectomy-An Initial Experience of 25 Cases with a New Technique. World J Lap Surg 2018;11(2):64-67.

Source of support: Nil

Conflict of interest: None

\section{BACKGROUND}

In Nepal, it is quite common to find patients with a large stone burden and thick gallbladder wall which often leads to specimen extraction difficulties. Out of all the available methods to facilitate the extraction like fascial dilatation, stone crushing, high-speed ultrasonic rotary, or laser lithotripsy, we prefer to use incision extension since it has been described as the optimal method and does not aggravate postoperative

General Surgeon

Department of Surgery, National Health Service, London, UK

Corresponding Author: Aswini Misra, General Surgeon, Department of Surgery, National Health Service, London, UK, e-mail: draswini@gmail.com pain. ${ }^{1}$ Many of the $11 \mathrm{~mm}$ epigastric wounds land up in a dimension of 13 to $14 \mathrm{~mm}$ or more at times at the completion of the procedure. However, we have used this wound extension to our advantage by introducing another $5 \mathrm{~mm}$ port through the epigastric wound from the outset. This not only obviates the need for any additional port insertion but also aids in specimen extraction. This forms the rationale behind two port laparoscopic cholecystectomy. With the technique described in this article, one will be able to perform laparoscopic cholecystectomy with only two incisions leading to a more cosmetic scar and less postoperative pain. Last decade has seen many innovations like squamous intraepithelial lesion (SILs), NOTES from healthcare industries driven by an ever-increasing demand for cosmesis. However, the cost factor keeps them out of the reach of a common man in developing countries. This technique certainly adds to cosmesis still fitting to the budget of a common man.

\section{PATIENTS AND METHODS}

Twenty-five patients underwent the operation from 2008 to 2010 after the hospital ethical committee approval. Informed consent was obtained from all the patients. The same team of surgeons performed all the surgeries. Every single patient had investigation proven gallstone or related complications. Operative time, hospital stay and complications were recorded in each case.

The patient characteristics are mentioned below. There were 10 male and 15 female patients, and none of the patients had any abdominal surgery in the past. The mean age was 40.5 years (range $27-55$ years). All the patients had body mass index (BMI) below 30. Total fourteen patients were anesthetic risk assessment (ASA) I and II were ASA II (8 patients were controlled hypertensives and 3 were controlled diabetics).

\section{Operative Technique}

The open technique does a peritoneal entry with the insertion of a $10 \mathrm{~mm}$ port through the umbilicus. After creating pneumoperitoneum, a 1-centimeter transverse skin incision is taken in the midline at a level 1 inch cephalad to the level of the inferior border of liver for the epigastric port. A $10 \mathrm{~mm}$ port is inserted through the 


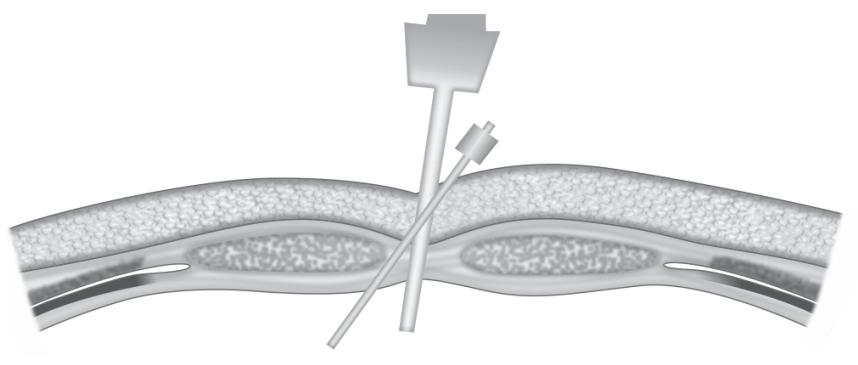

Fig. 1: Position of port assembly in the epigastric region

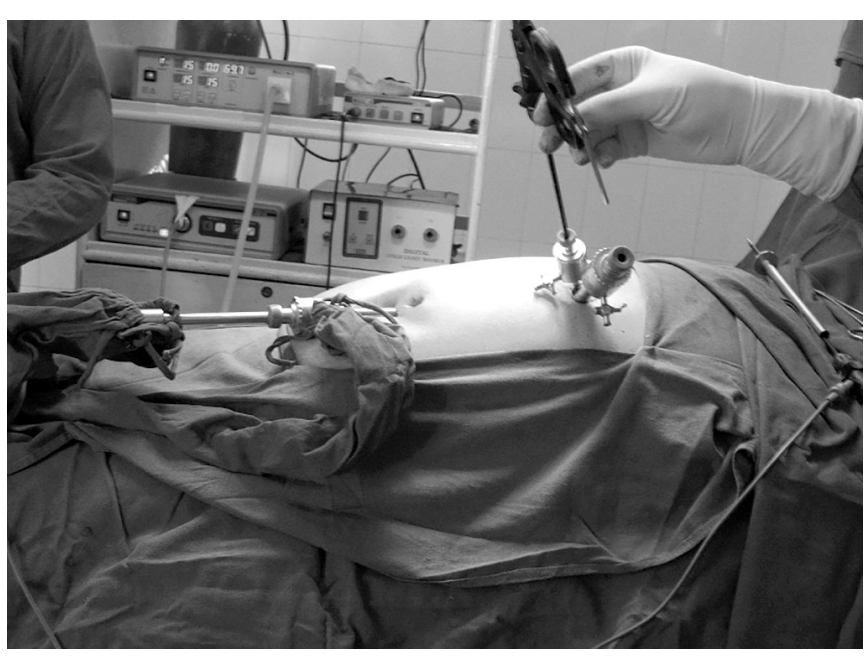

Fig. 3: Side view of the ports positions and port assembly

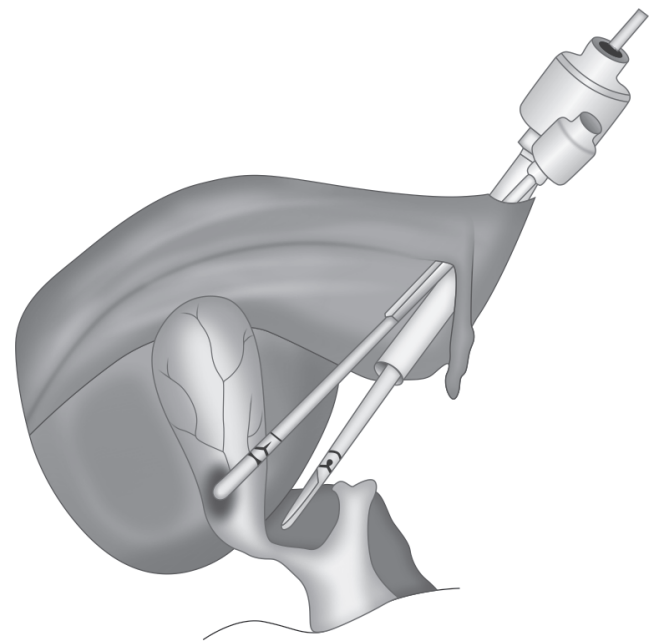

Fig. 5: Calot's triangle dissection using the port assembly

later incision vertically till it pierces the rectus sheath (This will be referred henceforth as port-2). Afterward, a slight right side angling of the port is done to bring it through the angle between the falciform ligament and the anterior peritoneum. A $5 \mathrm{~mm}$ grasper (with reducer) is introduced through the port 2 , and the fundus of the gallbladder is grasped, and traction is applied towards the right shoulder. This step displays the gallbladder anatomy in entirety. Now an intraoperative assessment is done to determine if the two-port laparoscopic cholecystectomy can be done safely (patient suitability has been described in the discussion). If conditions are found to be favorable, with the traction maintained in

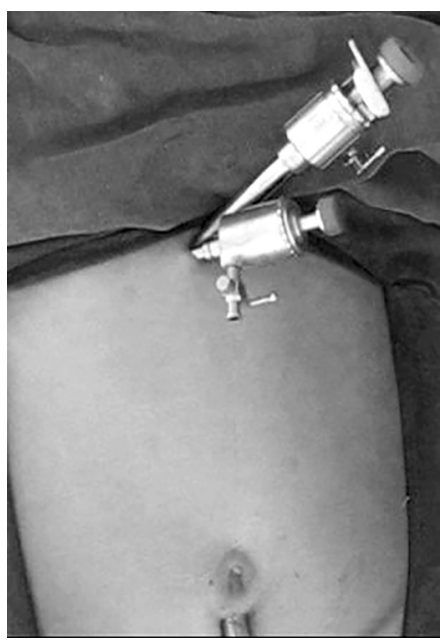

Fig. 2: Position of port assembly in the epigastric region-intraoperative view

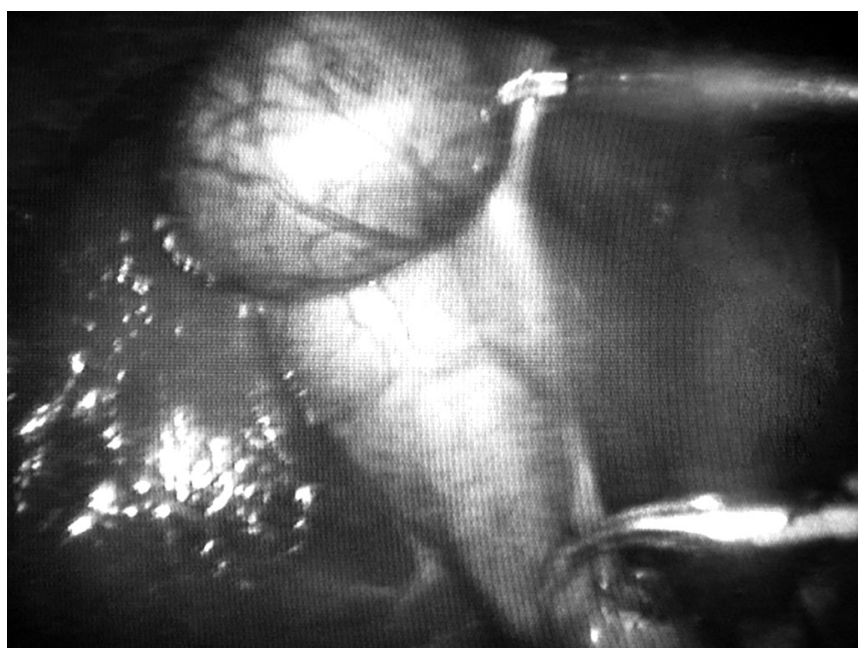

Fig. 4: Intraoperative view of gallbladder dissection

the described way, a $5 \mathrm{~mm}$ port is inserted through the existing epigastric skin incision (but through a separate stab traversing a different path to the peritoneal cavity) little away from the port two pointing towards the Hartman's pouch of the gallbladder (This will be referred henceforth as the port-3) (Figs 1 to 4). Before this step, the skin incision may be extended 3 to $5 \mathrm{~mm}$ or more as required.

Now appropriate traction is applied to the Hartman's pouch in the lateral direction by the port- 3 instrument, and this widens up the Calot's triangle. With a suitable instrument (preferably a Maryland introduced through the port-2), Calot's triangle dissection is done. The traction and dissection instruments are used interchangeably through the ports 2 and 3 as per requirement. The rotational freedom of the port three around port 2 helps in traction and dissection to be done at various points and depth (However the rotation of the port should never be attempted with the instrument inside the port) (Figs 4 and 5). The cystic artery and duct are circumferentially skeletonized. With double clips placed on the body side and a single clip on the specimen side, both the structures 
are divided. This step is completed by traction through the port 3 instrument and clip application through porttwo. With continued traction applied to the Hartman's pouch in the upward and right direction (this open up the interface between the gallbladder and the gallbladder fossa of the liver), the gallbladder is separated from the gallbladder fossa by electro-dissection with an appropriate instrument (either a monopolar hook, Maryland or scissor). Before the final detachment of gallbladder from the liver, the hemostasis of the gallbladder bed is achieved, and the cystic pedicle (artery and duct) security is confirmed. The $5 \mathrm{~mm}$ port is now withdrawn and the specimen extracted through the epigastric port. A generous amount of peritoneal wash is given, and $100 \mathrm{~mL}$ of normal saline mixed with bupivacaine is left in the subdiaphragmatic space. Pneumoperitoneum is evacuated, and the wounds closed in two layers.

Because of the presence of two ports in the same wound the range of their movement is likely to be affected. Hence, careful attention should be paid to proper alignment of the ports at the epigastric site. The chamber of the $5 \mathrm{~mm}$ port should be as close to the skin as possible whereas that of $10 \mathrm{~mm}$ port should be as far away from the skin as possible (Figs 2 and 5). The maneuverability and the freedom of a port depend on the rotational capacity or the swing of the ports (Please watch the video). With the measures mentioned above, we have observed that there is adequate overall maneuverability including a range of movement and reach of the instrument to complete the procedure safely. The right and left-hand instruments work in close harmony as an assembly, with one grasping/retracting at a short distance from the other one(Figs 4 and 5). They move in tandem performing the dissection bit by bit sequentially from Calot's Triangle to the fundus till the point of complete separation of the organ.

\section{RESULTS}

There was no incidence of the bile duct or vascular injury, bile leak, iatrogenic injury, intra-operative perforation of the gallbladder, bile spillage, significant procedural blood loss, significant gas leak or subcutaneous emphysema at either port site. The mean operating time was 50 minutes (range 40-155 minutes).

We have converted three cases from the two port technique to the standard four-port technique. One was due to the technical difficulty arising out of bleeding and the other two due to difficult intra-operative findings. These two cases had dense adhesions in the Calot's triangle and gallbladder fossa respectively. However, none of them required conversion to open cholecystectomy.

Patients were allowed orally as early as 6 hours following surgery. All patients were routinely discharged

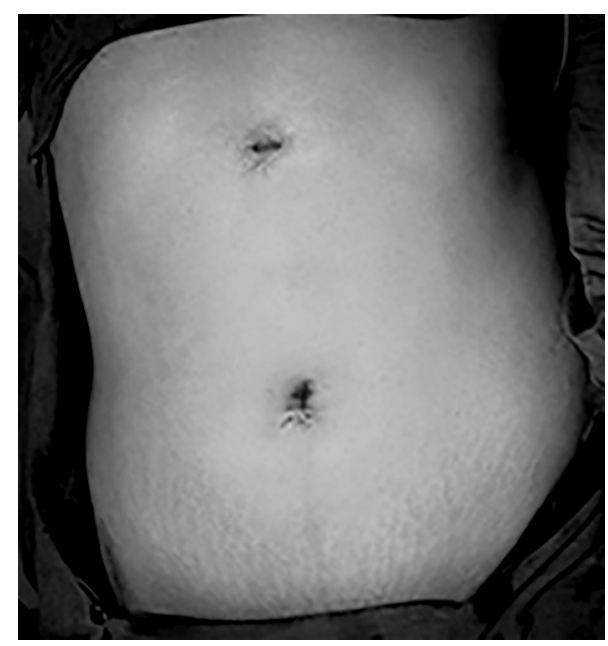

Fig. 6: Appearance of wounds immediately after closure

on the 2nd postoperative day except for two patients. One had severe abdominal pain and later developed surgical site infection, which subsided with wound drainage and the other patient developed fever in the postoperative period. All the patients were happy and satisfied due to rapid and comfortable recovery and of course, about their small wound. Many patients were astonished small incision used to perform the surgery and hence were curious to know the procedure details (Fig. 6). Patients were advised follow up on the 10th day, 3 months and 1 year following surgery. Out of 25 patients, 23 patients visited the hospital for 10th day follow-up and were fine at that point in time. However, only seven have completed three months follow up at the point of data collection, and none of them had any complications including port site hernia.

\section{DISCUSSION}

Although laparoscopic cholecystectomy has been practiced as a day care surgery, it is far from reality in our set-up as most of the patients are from remote rural and hilly areas with poor access to health care. That is the reason for the patient being discharged routinely on the 2nd postoperative day. Secondly, the follow-up of the patients has remained far from ideal. Many of them, once discharged, tend to avoid hospital follow up unless they are unwell. The geographic and telecommunication barriers are other factors which have prevented us from reaching out to them.

Two-port laparoscopic cholecystectomy has been practiced by many surgeons successfully and has been reported to be safe and superior to 4 port cholecystectomy in terms of pain, cosmesis and patient acceptance. ${ }^{2,3}$ Various techniques and special instruments like innovative extracorporeal knot by Mishra et al., "Twin-port” system (that allows a $5 \mathrm{~mm}$ camera and a forceps through a single port) by Kagaya et al., $2 \mathrm{~mm}$ or $3 \mathrm{~mm}$ endo graspers by Lee KW, have been used to accomplish the procedure without the 
need of additional ports. However, traction sutures on gallbladder may end up in tearing of the organ leading to stone spillage and associated consequences like an abscess, fistula formation and other septic complications later on. ${ }^{4-7}$ This possibility further increases in patients with a high stone burden. So, we aim at the gentle handling of the gallbladder and take preventive steps to avoid intra-operative spillage and hence do not use sutures for traction. ${ }^{8,9}$ However, the present technique requires no special instrument or complex technique.

Although the present technique is safe, there are some inherent limitations. This should not be used for cases where technical difficulty is anticipated or encountered for example in acute cholecystitis, empyema, dense adhesions in Calot's triangle, intrahepatic gallbladder, anatomic abnormality in the hepato-biliary system, Mirrizzi's syndrome, cirrhosis of the liver, etc. Drain insertion in the subcostal region nullifies all the purported advantages of the procedure. Hence, it is better to perform a feasibility assessment before attempting this two port technique, and difficult cases should routinely be done in four port fashion. If there is bleeding during the procedure, a low threshold should be maintained to convert to the standard four-port technique. Meticulous dissection and gentle handling of instruments are a sine qua non for safe and successful completion of the procedure. One should not expect the freedom of a four-port technique in this method. With careful case selection coupled with precise technique and patience, one can make this twoport laparoscopic cholecystectomy an amazing reality in one's surgical practice.

\section{REFERENCES}

1. Bordelon BM, Hobday KA, Hunter JG. Incision extension is the optimal method of difficult gallbladder extraction at laparoscopic cholecystectomy. Surg Endosc. 1992 Sep-Oct; 6(5):225-227.

2. Shimura T, Suehiro T, Suzuki H, Mochida Y, Okada K, Araki $\mathrm{K}$, Kuwano H. Laparoscopic cholecystectomy using a novel two-port technique for surgical residency training. Int Surg. 2009 Apr-Jun;94(2):149-153.

3. Poon CM, Chan KW, Lee DW, Chan KC, Ko CW, Cheung $\mathrm{HY}$, et al. Two-port versus four-port laparoscopic cholecystectomy. Surgical Endoscopy and Other Interventional Techniques. 2003 Oct 1;17(10):1624-1627.

4. Hougård K, Bergenfeldt M. Abdominal fistula 7 years after laparoscopic cholecystectomy. Ugeskrift for laeger. 2008 Sep;170(36):2803.

5. Arishi AR, Rabie ME, Khan MS, Sumaili H, Shaabi H, Michael NT, et al. Spilled gallstones: the source of an enigma. JSLS: Journal of the Society of Laparoendoscopic Surgeons. 2008 Jul;12(3):321-325.

6. Bhati CS, Tamijmarane A, Bramhall SR. A tale of three spilled gall stones: one liver mass and two abscesses. Dig Surg. 2006;23(3):198-200.

7. Patterson EJ, Nagy AG. Don't cry over spilled stones? Complications of gallstones spilled during laparoscopic cholecystectomy: case report and literature review. Can J Surg. 1997 Aug;40(4):300-304.

8. Waqar SH, Shah SF, Khan IA, Ch TS, Abdullah MT, Malik ZI, et al. Two-port laparoscopic cholecystectomy-a new technique. J Ayub Med Coll Abbottabad. 2008 Oct- Dec;20(4):167168.

9. Sathesh-Kumar T, Saklani AP, Vinayagam R, Blackett RL. Spilled gall stones during laparoscopic cholecystectomy: a review of the literature. Postgraduate medical journal. 2004 Feb 1;80(940):77-79. 